\title{
Effect of Increasing Body Mass Index on Obstructive Sleep Apnea in Children
}

\author{
Katharina D. Graw-Panzer*, Hiren Muzumdar, Supriya Jambhekar, Nira A. Goldstein and Madu Rao
}

Division of Pediatric Pulmonology, SUNY Downstate Medical Center, 450 Clarkson Avenue, Box 49, Brooklyn, NY 11203, USA

\begin{abstract}
Obesity is associated with obstructive sleep apnea in the adult and pediatric population. The aim of our study was to assess the association between increasing body mass index on the severity of obstructive sleep apnea in children.

We reviewed retrospectively 740 children with symptoms suggestive of obstructive sleep apnea who underwent a sleep study at an inner city university hospital. Obstructive sleep apnea was defined as respiratory disturbance index of 5 per hour or more. Statistical analysis included chi square test and regression analysis.

The results were as follows: 740 children, $56 \%$ were male. Mean age $( \pm$ SD) $6.0( \pm 3.4)$ years. $66 \%$ were 6 years of age and younger and $34 \%$ were 7 years of age and older. $61 \%$ had a nap study positive for obstructive sleep apnea (63\% of children $\leq 6$ years and $69 \% \geq 7$ years). Mean body mass index z-score was $+1.1( \pm 1.5)(+1.0( \pm 1.6)$ in children $\leq 6$ years and $+1.5( \pm 1.2) \geq 7$ years). In children 7 years of age and older there was a relationship between body mass index z-score and respiratory disturbance index $(r=0.188, p=0.003)$. In children 6 years of age and younger there was no association. There was no relationship between body mass index and desaturation.

Increasing body mass index is associated with increasing severity of obstructive sleep apnea in children 7 years of age and older.
\end{abstract}

Keywords: Body mass index, child, obesity, obstructive sleep apnea, overweight, sleep study.

\section{INTRODUCTION}

Obstructive sleep apnea (OSA) in children is a disorder of breathing during sleep characterized by prolonged partial upper airway obstruction and/or intermittent complete obstruction that disrupts normal ventilation during sleep and normal sleep patterns. It is associated with symptoms including habitual snoring, sleep difficulties, and/or daytime neurobehavioral problems. Complications may include growth abnormalities, neurological disorders, and cor pulmonale, especially in severe cases [1-3]. Anatomic narrowing and neuromuscular abnormalities cause disturbance of airflow during sleep. The vast majority of children with OSA have large tonsils and adenoids, and improve after adenotonsillectomy [4]. In obese children the effect of fat deposition in the pharyngeal muscles and extrapharyngeal compression from superficial subcutaneous fat decreases the pharyngeal lumen, predisposing to obstruction [5]. OSA is common in pediatrics, affecting 1 to $3 \%$ of all children [6-9]. In obese children the prevalence has been reported from $5.7 \%$ to $36 \%$ [10,11]. The risk of having OSA has been estimated to be 4.6 times higher (OR 4.59; 95\% confidence interval (CI), 1.58 to 13.33) in the presence of obesity [9].

The prevalence of overweight among US children is increasing. Approximately $15.3 \%$ of children (ages 6 to 11 years) and $15.5 \%$ of adolescents (ages 12 to 19 years) are

*Address correspondence to this author at the Division of Pediatric Pulmonology, SUNY Downstate Medical Center, 450 Clarkson Avenue, Box 49, Brooklyn, NY 11203, USA; Tel: 718-270-1647; Fax: 718-2701985; E-mail: Katharina.Graw-Panzer@downstate.edu overweight [12]. The prevalence of overweight quadrupled over 25 years among children and more than doubled over 25 years among adolescents [12].

Studies have shown an association between obesity and OSA in children $[9-11,13,14]$. The objective of this study was to assess the association between increasing body mass index (BMI) on the severity of OSA in children.

\section{MATERIALS AND METHODOLOGY}

We recorded the data of children who underwent nap polysomnography (PSG) for suspected OSA from 1995 to 2004. The children were referred by their primary care providers and ear-nose-throat specialist with symptoms suggestive of OSA. The study was performed at the Children's Hospital at Downstate, State University of New York Downstate College of Medicine. We obtained the information by reviewing the medical records retrospectively. All records of children 2 years of age and above with data on weight and height were included.

Daytime nap PSG were performed and monitored at the Children's Hospital sleep laboratory by a pulmonary technician skilled in pediatric PSG by the EdenTec ${ }^{\circledR}$ system (EdenTec Corporation, $\mathrm{MN}$ ) in a quiet, dark room at an ambient temperature of $24^{\circ} \mathrm{C}$. The children went to sleep spontaneously after sleep deprivation the night before the study. The sleep state was confirmed by the technician who performed the PSG. A minimum of one hour of sleep was recorded. The following parameters were simultaneously and continuously measured: oro-nasal airflow by thermistor (BreathSensor ${ }^{\mathrm{TM}}$, Nellcor, MN), arterial oxygen saturation 
by pulse oximetry (OxiMax ${ }^{\mathrm{TM}}$, Nellcor, MN), chest wall movements and heart rate (Meditrace ${ }^{\circ}$, Kendall, MA). The following parameters were evaluated: apnea, defined as complete cessation of air flow at nose and mouth for at least 10 seconds, hypopnea, defined as a $50 \%$ reduction of airflow for at least 10 seconds with a $3 \%$ drop in oxygen saturation, respiratory disturbance index (RDI) measured as number of apneas and hypopneas divided by the hours of sleep and desaturation defined as mean oxygen saturation less than $92 \%$ [15]. OSA was defined as RDI of 5 per hour or more [16].

Height and weight were measured with standard medical equipment. BMI was calculated as weight $(\mathrm{kg})$ divided by the square of the height in meters $\left(\mathrm{m}^{2}\right)$, and was further analyzed as BMI z-scores. Age and gender adjusted BMI zscores were obtained from the National Center for Health Statistics (NCHS) 02/2005 database. Overweight is defined as at or above the 95th percentile of BMI for age [17].

The data was analyzed as a whole and also as grouped into younger and older children. The cutoff was chosen at 6 years because adenotonsillar hypertrophy peaks in the preschool age [18].

The Institutional Review Board of Downstate Medical Center at which the study was performed exempted the study from review under the guideline 45CFR46.101(b)(4).

\section{Statistical Analyses}

Statistical analysis of proportions comparing groups of children was done by chi square test. The relationships between BMI z-score to RDI and desaturation were evaluated by linear regression analysis, prediction of OSA from BMI $\mathrm{Z}$-score by simple prediction model and interaction between variables with multivariable analysis. A p value of $<0.05$ was considered statistically significant. All analyses were performed using SPSS (version 17) and SAS (version 9.3).

\section{RESULTS}

Data on 740 children were analyzed. Table 1 presents the demographic characteristics. $56 \%$ were boys and $44 \%$ girls. The study population was predominantly African-American and Hispanic. Mean age ( \pm standard deviation) was $6.0( \pm$ 3.4 ) years (range $2-18$ years). Mean BMI z-score was +1.1 $( \pm 1.5) .40 \%$ of the children were overweight. Mean RDI was $17.6( \pm 20.3)$. OSA was diagnosed in $65 \%$ of children.

$66 \%$ of children were 6 years of age and younger, and $34 \% 7$ years of age and older. There were more boys than girls in both age groups. The mean BMI z-score was significantly higher in the group of children 7 years of age and older $(+1.5$ versus $+1.0, p<0.001) .51 \%$ of children 7 years of age and older were overweight compared to $35 \%$ of children 6 years of age and younger.

OSA was diagnosed in $63 \%$ of children 6 years of age and younger and in $69 \%$ of children 7 years of age and older.

There was a significant interaction of BMI z-score by continuous age $(\mathrm{p}=0.041)$. As age increases, BMI z-score becomes a stronger and stronger predictor of OSA (age 4 years: OR 1.095 ; $95 \% \mathrm{CI}, 0.405$ to 2.959 ; age 10 years: OR 1.142 ; $95 \% \mathrm{CI}, 0.106$ to12.328; age 16 years: OR 1.192; 95 $\% \mathrm{CI}, 0.028$ to 51.508 . In children 7 years of age and older there was an association between increasing BMI z-score and RDI ( $\mathrm{r}=0.188, \mathrm{p}=0.003$ ) (Fig. 1), but not in children 6 years of age and younger $(r=0.032, p=0.481)$ (Fig. 2). The prediction of OSA from BMI z-score (for each BMI z-score increase by one unit) did not meet significance: OR 1.005; $95 \% \mathrm{CI}, 0.910$ to $1.111, \mathrm{p}=0.918$. No association could be

Table 1. Demographic Characteristics and Results

\begin{tabular}{|c|c|c|c|c|}
\hline Variables & All $(n=740)$ & Age $\leq 6$ yrs $(n=486)$ & Age $\geq 7$ yrs $(n=254)$ & p value ${ }^{a}$ \\
\hline Gender, male, No (\%) & $416(56)$ & $278(57)$ & $138(54)$ & 0.6 \\
\hline Age, years & $6.0(3.4)$ & $4.0(1.4)$ & $9.9(2.8)$ & $<0.001$ \\
\hline Race $^{\mathrm{b}}$, African-American, No (\%) & $435(70)$ & $285(72)$ & $150(68)$ & 0.3 \\
\hline Race $^{\mathrm{b}}$, Hispanic, No (\%) & $149(24)$ & $90(23)$ & $59(27)$ & 0.3 \\
\hline Race $^{\mathrm{b}}$, Asian, No (\%) & $22(4)$ & $13(3)$ & $9(4)$ & 0.6 \\
\hline Race $^{\mathrm{b}}$, Caucasian, No (\%) & $14(2)$ & $10(3)$ & $4(2)$ & 0.6 \\
\hline BMI z-score & $+1.1 \pm 1.5$ & $+1.0 \pm 1.6$ & $+1.5 \pm 1.2$ & $<0.001$ \\
\hline Overweight $^{\mathrm{c}}$, No (\%) & $299(40)$ & $170(35)$ & $129(51)$ & $<0.001$ \\
\hline RDI & $17.6(20.3)$ & $16.6(19.0)$ & $19.3(22.4)$ & 0.08 \\
\hline $\mathrm{OSA}^{\mathrm{d}}$, No $(\%)$ & $481(65)$ & $305(63)$ & $176(69)$ & 0.08 \\
\hline OSA and overweight, No (\%) & $255 / 481(53)$ & $133 / 305(44)$ & $122 / 176(69)$ & $<0.001$ \\
\hline Desaturation $^{\mathrm{e}}$, No (\%) & $183(25)$ & $113(23)$ & $70(28)$ & 0.2 \\
\hline
\end{tabular}

Continuous variables are presented as mean \pm standard deviation.

${ }^{a} \mathrm{p}$ value for comparison of children $\leq 6$ yrs to $\geq 7 \mathrm{yrs}$.

${ }^{\mathrm{b}}$ Information about race in 620 children $(\leq 6$ yrs: $\mathrm{n}=398, \geq 7$ yrs: $\mathrm{n}=222)$.

'Overweight is defined as at or above the 95 th percentile of BMI for age.

${ }^{\mathrm{d}}$ Obstructive sleep apnea (OSA) defined as respiratory disturbance index (RDI) $\geq 5$.

${ }^{\mathrm{e}}$ Desaturation defined as mean oxygen saturation less than $92 \%$. 
established between BMI z-score and oxygen desaturation ( $\leq 6$ yrs: $r=0.04, p=0.3, \geq 7$ yrs: $r=0.06, p=0.3$ ).

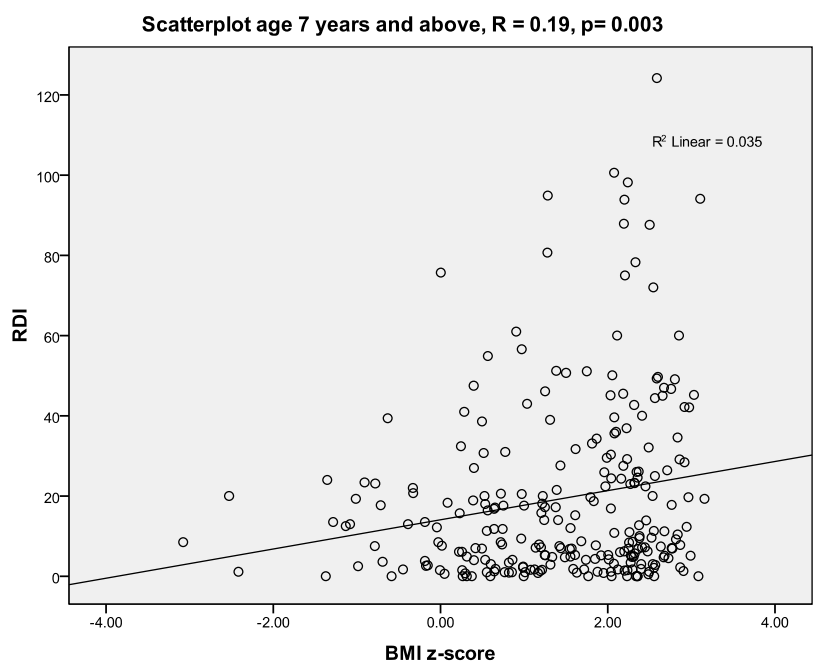

Fig. (1). In children 7 years of age and older there was an association between $\mathrm{BMI}$ z-score and RDI.

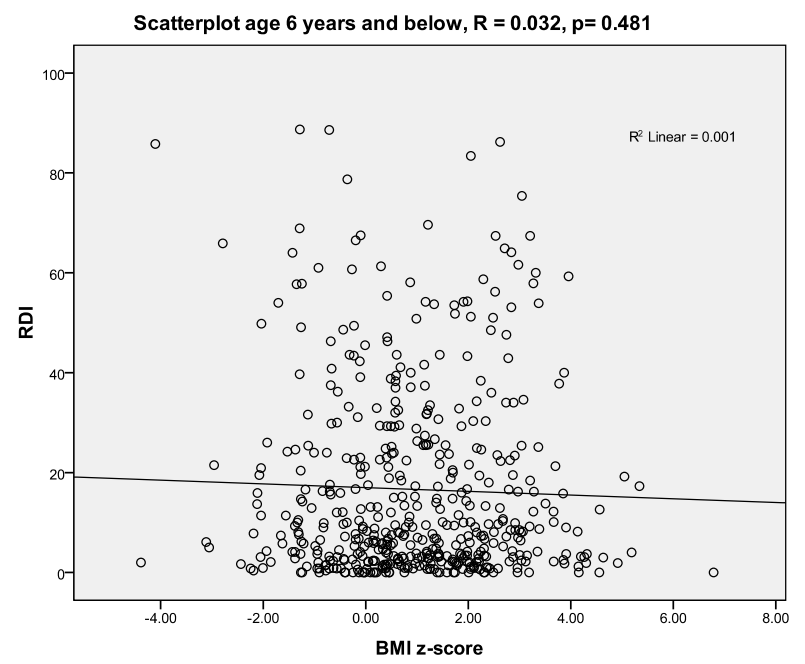

Fig. (2). In children 6 years of age and below there was no association between BMI z-score and RDI.

\section{DISCUSSION}

In the review of our data on children with symptoms of OSA we found an interaction between age and BMI z-score explaining OSA. With increasing age, BMI z-score becomes a stronger and stronger predictor of OSA. In children 7 years of age and older increasing BMI z-score was associated with increasing severity of OSA. There was no association between BMI z-score and RDI in children 6 years of age and younger.

Other studies have also shown an association of obesity with OSA in children. Marcus et al. [10] reported that 36\% of 22, predominantly African-American obese children, mean age $10 \pm 5$ years who were not selected based on symptoms of sleep disordered breathing had abnormal PSGs. Obesity was defined as body weight $>120 \%$ of their ideal body weight (IBW) or skin fold thickness $>85$ th percentile. There was a positive correlation between the degree of obesity and apnea index $(r=0.47, \mathrm{p}<0.05)$. Silvestri et al. [13] reported obstructive apnea in 59\% of 32 obese children, mean age $8.6 \pm 3$ years with a history of sleep associated breathing disorders. Obesity was defined as weight $>95$ th percentile, $>120 \%$ of IBW and BMI >90th percentile. There was no association between any polysomnographic measurements and percent of IBW. However by applying discriminate analysis polysomnographic abnormalities could be predicted with up to $81 \%$ reliability in very obese children ( $\geq 200 \%$ IBW). Redline et al. [9] studied 399 children from families of index cases with sleep apnea, mean age $11.1 \pm$ 4.3 years, and controls. They found that obese children (defined by a $\mathrm{BMI}>28, \mathrm{n}=38$ ) were four to five times more likely to have sleep disordered breathing (SDB), than were nonobese children. Further, for each increase of $1 \mathrm{~kg} / \mathrm{m}^{2}$ of BMI above the mean, the risk of SDB increased by $12 \%$. Wing et al. [14] compared 46 obese children mean age 10.8 \pm 2.3 years with 44 matched normal weight children, all not selected based on symptoms of SDB. Obesity was defined by weight $\geq 120 \%$ IBW. They found that SDB was significantly related to BMI (OR $1.20,95 \%$ CI 1.08 to $1.33, \mathrm{p}=0.001$ ). $33 \%$ of obese compared to $5 \%$ of nonobese children had and RDI $\geq 5$. On the other hand Mallory et al. [19] studied 41 obese children (defined by $>150 \%$ IBW), mean age $10.3 \pm$ 4.4 years referred with histories suggestive of SDB. They demonstrated no significant association between percent IBW and any abnormality on PSG. OSA was diagnosed in $37 \%$ of children.

In all the studies different sleep study methods, definition of OSA and obesity have been applied and hence make a direct comparison difficult. In contrast to the cited studies, we analyzed the data obtained on sleep studies performed on a large cohort of children with BMI ranging across the normal to overweight spectrum that had symptoms of SDB and found an age dependent association of BMI with OSA. We speculate that in the preschool age group, when the majority of cases of OSA are associated with adenotonsillar hypertrophy, the effect of high BMI may be minor compared to the influence of adenotonsillar hypertrophy.

Our study has some limitations. Sleep was recorded during daytime naps rather than overnight PSG. It has been shown that nap studies are a good screening tool for OSA. Marcus et al. [20] demonstrated that the sensitivity of nap study compared to overnight PSG is $73.7 \%$, the specificity $100 \%$ and the positive predictive value $100 \%$. Reliance on data from nap studies may have led to an underestimation of OSA. As all patients were studied with nap PSG we postulate that the limitation was equal in younger and older, overweight and non overweight children and the eventual comparison between the groups would have been the same even if overnight PSG was used. Saeed et al. [21] showed that there was no significant difference in the apnea index comparing nap study with PSG in 143 children.

Our study was done on children referred with symptoms of OSA, leading to a high rate of abnormal sleep studies and 
does not provide an estimate of the incidence of OSA in the general overweight pediatric population. The high rate of OSA and the high rate of obesity in our study population may have obscured the relationship between OSA and BMI and may not apply to the general population of children.

We studied an inner-city population which may not represent other areas of the US. The study population was predominantly African-American (70\%). Black children are more likely to have SDB [22], the basis for racial differences is not clear, but may be explained by craniofacial risk factors [23] or genetic predisposition [24].

The duration of an apneic event was defined as $\geq 10$ seconds rather than the current consensus of 2 respiratory cycles. The data collection started at a time when apnea was defined as $\geq 10 \mathrm{sec}$, for standardization the definition of apnea length was not changed during the period of data collection. This may have underreported the prevalence of OSA in our group of patients. Again, as the same definition was used for all studies this should not affect the comparison between sub-groups. The current recommended sensor for detection of airflow for identification of hypopnea is a nasal air pressure transducer and the alternate sensor is an oronasal thermal sensor [25], which was used in our study. Studies comparing both technologies have found that nasal pressure measurement was more sensitive than thermistry especially in detecting hypopneas [26-28]. In addition this is a retrospective review of patients and did not have a control group for comparison.

\section{CONCLUSION}

Our study looks at the association between overweight and OSA in children clinically suspected to have OSA. We found that with increasing age, BMI z-score becomes a stronger and stronger predictor of OSA. Increasing BMI correlates with increasing severity of OSA in children 7 years of age and older.

\section{ACKNOWLEDGEMENTS}

We thank Dr. Jeremy Weedon, PhD, from the Scientific Academic Computing Center, State University of New York at Brooklyn, NY, for assistance with the statistical analysis, Ms. Mina Iman for technical support, Yuri Rothshteyn and Geeta Malviya, MBBS, for data collection.

\section{REFERENCES}

[1] Guilleminault C, Winkle R, Korobkin R, Simmons B. Children and nocturnal snoring: evaluation of the effects of sleep related respiratory resistive load and daytime functioning. Eur J Pediatr 1982; 139(3): 165-71.

[2] Tal A, Leiberman A, Margulis G, Sofer S. Ventricular dysfunction in children with obstructive sleep apnea: radionuclide assessment. Pediatr Pulmonol 1988; 4(3): 139-43.

[3] Marcus CL, Greene MG, Carroll JL. Blood pressure in children with obstructive sleep apnea. Am J Respir Crit Care Med 1998; 157(4 Pt 1): 1098-103.

[4] Brietzke SE, Gallagher D. The effectiveness of tonsillectomy and adenoidectomy in the treatment of pediatric obstructive sleep apnea/hypopnea syndrome: a meta-analysis. Otolaryngol Head Neck Surg 2006; 134(6): 979-84.

[5] Shelton KE, Woodson H, Gay S, Suratt PM. Pharyngeal fat in obstructive sleep apnea. Am Rev Respir Dis 1993; 148(2): 462-6.

[6] Gislason T, Benediktsdottir B. Snoring, apneic episodes, and nocturnal hypoxemia among children 6 months to 6 years old. An epi- demiologic study of lower limit of prevalence. Chest 1995; 107(4): 963-6.

[7] Ali NJ, Pitson DJ, Stradling JR. Snoring, sleep disturbance, and behaviour in 4-5 year olds. Arch Dis Child 1993; 68(3): 360-6.

[8] Kaditis AG, Finder J, Alexopoulos EI, et al. Sleep-disordered breathing in 3,680 Greek children. Pediatr Pulmonol 2004; 37(6): 499-509.

[9] Redline S, Tishler PV, Schluchter M, Aylor J, Clark K, Graham G. Risk factors for sleep-disordered breathing in children. Associations with obesity, race, and respiratory problems. Am J Respir Crit Care Med 1999; 159(5 Pt 1): 1527-32.

[10] Marcus CL, Curtis S, Koerner CB, Joffe A, Serwint JR, Loughlin GM. Evaluation of pulmonary function and polysomnography in obese children and adolescents. Pediatr Pulmonol 1996; 21(3): 17683.

[11] Chay OM, Goh A, Abisheganaden J, et al. Obstructive sleep apnea syndrome in obese Singapore children. Pediatr Pulmonol 2000; 29(4): 284-90.

[12] Ogden CL, Flegal KM, Carroll MD, Johnson CL. Prevalence and trends in overweight among US children and adolescents, 19992000. JAMA 2002; 288(14): 1728-32.

[13] Silvestri JM, Weese-Mayer DE, Bass MT, Kenny AS, Hauptman SA, Pearsall SM. Polysomnography in obese children with a history of sleep-associated breathing disorders. Pediatr Pulmonol 1993; 16(2): 124-9.

[14] Wing YK, Hui SH, Pak WM, et al. A controlled study of sleep related disordered breathing in obese children. Arch Dis Child 2003; 88(12): 1043-7.

[15] Marcus CL, Omlin KJ, Basinki DJ, et al. Normal polysomnographic values for children and adolescents. Am Rev Respir Dis 1992; 146(5 Pt 1): 1235-9.

[16] Guilleminault C, Korobkin R, Winkle R. A review of 50 children with obstructive sleep apnea syndrome. Lung 1981; 159(5): 27587.

[17] Kuczmarski RJ, Ogden CL, Grummer-Strawn LM, et al. CDC growth charts: United States. Adv Data 2000; 314: 1-27.

[18] Jeans WD, Fernando DC, Maw AR, Leighton BC. A longitudinal study of the growth of the nasopharynx and its contents in normal children. Br J Radiol 1981; 54(638): 117-21.

[19] Mallory GB Jr., Fiser DH, Jackson R. Sleep-associated breathing disorders in morbidly obese children and adolescents. J Pediatr 1989; 115(6): 892-7.

[20] Marcus CL, Keens TG, Ward SL. Comparison of nap and overnight polysomnography in children. Pediatr Pulmonol 1992; 13(1): 16-21.

[21] Saeed MM, Keens TG, Stabile MW, Bolokowicz J, Davidson Ward SL. Should children with suspected obstructive sleep apnea syndrome and normal nap sleep studies have overnight sleep studies? Chest 2000; 118(2): 360-5.

[22] Rosen CL, Larkin EK, Kirchner HL, et al. Prevalence and risk factors for sleep-disordered breathing in 8- to 11-year-old children: association with race and prematurity. J Pediatr 2003; 142(4): 3839.

[23] Cakirer B, Hans MG, Graham G, Aylor J, Tishler PV, Redline S. The relationship between craniofacial morphology and obstructive sleep apnea in whites and in African-Americans. Am J Respir Crit Care Med 2001; 163(4): 947-50.

[24] Buxbaum SG, Elston RC, Tishler PV, Redline S. Genetics of the apnea hypopnea index in Caucasians and African Americans: I. Segregation analysis. Genet Epidemiol 2002; 22(3): 243-53.

[25] Iber C A-IS, Chesson A, Quan SF. for the american academy of sleep medicine. The AASM Manual for the Scoring of Sleep and Associated Events: Rules, Terminology and Technical Specifications. $1^{\text {st }}$ ed: Westchester, Illinois: Am Acad Sleep Med 2007.

[26] Budhiraja R, Goodwin JL, Parthasarathy S, Quan SF. Comparison of nasal pressure transducer and thermistor for detection of respiratory events during polysomnography in children. Sleep 2005; 28(9): 1117-21. 
[27] Serebrisky D, Cordero R, Mandeli J, Kattan M, Lamm C. Assessment of inspiratory flow limitation in children with sleepdisordered breathing by a nasal cannula pressure transducer system. Pediatr Pulmonol 2002; 33(5): 380-7.
[28] Trang H, Leske V, Gaultier C. Use of nasal cannula for detecting sleep apneas and hypopneas in infants and children. Am J Respir Crit Care Med 2002; 166(4): 464-8.

(C) Graw-Panzer et al.; Licensee Bentham Open .

This is an open access article licensed under the terms of the Creative Commons Attribution Non-Commercial License (http://creativecommons.org/licenses/by-nc/3.0/) which permits unrestricted, non-commercial use, distribution and reproduction in any medium, provided the work is properly cited. 